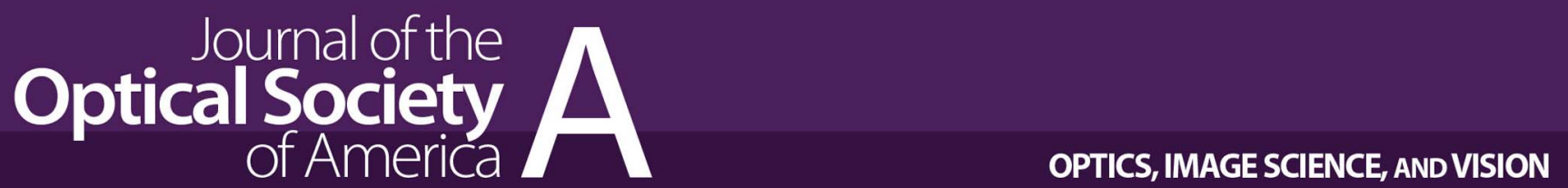

\title{
Visibility of sparkle in metallic paints
}

\author{
Eric Kirchner, ${ }^{1, *}$ Ivo van der Lans, ${ }^{1}$ Esther Perales, ${ }^{2}$ Francisco Martínez-Verdú, ${ }^{2}$ \\ Joaquín Campos, ${ }^{3}$ and Alejandro Ferrero ${ }^{3}$ \\ ${ }^{1}$ Color RD\&l, AkzoNobel Performance Coatings, Sassenheim, The Netherlands \\ ${ }^{2}$ Color \& Vision Group, University of Alicante, Carretera de San Vicente del Raspeig s/n 03690 Alicante, Spain \\ ${ }^{3}$ Instituto de Óptica, Consejo Superior de Investigaciones Científicas (CSIC), Madrid 28006, Spain \\ *Corresponding author: eric.kirchner@akzonobel.com
}

Received 13 November 2014; revised 13 March 2015; accepted 15 March 2015; posted 17 March 2015 (Doc. ID 226828 ); published 27 April 2015

\begin{abstract}
For suitable illumination and observation conditions, sparkles may be observed in metallic coatings. The visibility of these sparkles depends critically on their intensity, and on the paint medium surrounding the metallic flakes. Based on previous perception studies from other disciplines, we derive equations for the threshold for sparkles to be visible. The resulting equations show how the visibility of sparkles varies with the luminosity and distance of the light source, the diameter of the metallic flakes, and the reflection properties of the paint medium. The predictions are confirmed by common observations on metallic sparkle. For example, under appropriate conditions even metallic flakes as small as $\mathbf{1} \boldsymbol{\mu m}$ diameter may be visible as sparkle, whereas under intense spot light the finer grades of metallic coatings do not show sparkle. We show that in direct sunlight, dark coarse metallic coatings show sparkles that are brighter than the brightest stars and planets in the night sky. Finally, we give equations to predict the number of visually distinguishable flake intensities, depending on local conditions. These equations are confirmed by previous results. Several practical examples for applying the equations derived in this article are provided. (c) 2015 Optical Society of America
\end{abstract}

OCIS codes: (330.1710) Color, measurement; (330.1070) Vision - acuity; (330.1720) Color vision; (330.1800) Vision - contrast sensitivity; (330.6180) Spectral discrimination; (350.1260) Astronomical optics.

\section{INTRODUCTION}

The visual appearance of metallic coatings is characterized not only by their color, but also by texture. Under intense directional light, this texture shows up as a multitude of tiny but intense light spots that are brighter than surround, a phenomenon that is often referred to as sparkle [1]. There is a clear need to quantitatively describe how the perceived sparkle depends on parameters such as concentration and size of sparkling pigments and their contrast with the surrounding paint. Thus far, only qualitative relationships have been published $[2, \underline{3}]$, as well as a few crude estimations suggesting that the sparkle effect is strong for metallic flakes with average diameter exceeding 15 [్], 18

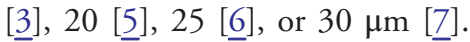

These estimations probably vary so widely because the visibility of sparkle can be expected to depend strongly on illumination and viewing conditions. Perception studies have shown that the perceived brightness of a stimulus depends critically on the luminance ratio between stimulus and its surround [8]. In this article, we will use existing literature from other disciplines to predict under which conditions light reflection from a metallic flake will lead to a perceived sparkle effect. The derived equations take into account the influence from illumination and observation conditions, as well as the relevant physical properties of the metallic flakes and the paint medium surrounding them.

The equations presented here may be useful for physical models that aim to convert photographic images of sparkle into sparkle measurements. For such models, it is necessary to mathematically distinguish sparkle pixels from surround $[\underline{9}, \underline{10}]$. Also, for rendering algorithms to generate realistic images of sparkle, it is necessary to account for sparkle as perceived by human observers $[11,12]$. The visibility of objects in natural waters [13] and the visibility of solar glitter on a ruffled sea [14-16] are other application areas of the present work.

\section{VISUAL THRESHOLD FOR SPARKLE INTENSITY}

The term sparkle intensity is widely used in the paint industry, and refers to its perceived brightness. However, intensity is a problematic quantity, since it has different definitions in different but related fields of optics [17]. Before discussing visual thresholds, we first need to clearly define the term sparkle intensity. 
The photometric quantity for the perceived brightness of an extended light source is luminance [17-20], with unit $\operatorname{lm} /\left(\mathrm{m}^{2} \cdot \mathrm{sr}\right)=\mathrm{cd} / \mathrm{m}^{2}$. When observing a sparkle within its surrounding, the surrounding paint forms an extended area of isotropic and uniform emission, for which the brightness is expressed as luminance.

However, for expressing the perceived brightness of a sparkle, luminance is not a practical quantity. This is caused by the size of the sparkle being so small that it cannot or can hardly be resolved by the human visual system or by a measurement device. Also, the size of the flake particle that causes the sparkle is usually not known. Since the solid angle subtended by the sparkle is usually not known, luminance cannot be calculated. Even in the case of highly directional illumination from a source with small angular size, and with flakes acting as mirrors that reflect the luminance of the source, the luminance cannot be used because the reflectance of the flake is often not known. Therefore, the perceived brightness of a (near) point-source like a sparkle is better represented by considering the total luminous flux per unit area on the observing position, a photometric quantity known as illuminance $\left[\mathrm{lm} / \mathrm{m}^{2}\right.$ or $\left.\mathrm{cd}\right]$.

Based on this analysis, we will use the term sparkle intensity to refer to the illuminance (notation symbol: $E$ ) of the optical radiation incident on the eye pupil, where this optical radiation originates from a light source and reflects from a metallic or pearlescent flake inside an effect coating before it reaches the eye of the observer.

We note that a similar situation occurs when expressing the brightness of light from a star. In order to avoid having to calculate the angular size of the star, the perceived brightness of light from stars is usually expressed as illuminance (unit: $\mathrm{lm} / \mathrm{m}^{2}$ ). In the astronomical literature it is common to express the perceived brightness of extended light emitters, such as the Sun or distant nebulas for which the angular size can be determined, in terms of luminance. Therefore, our choice to express the perceived sparkle intensity in terms of illuminance, and to use luminance for the perceived brightness of the paint area surrounding a sparkle, agrees with the astronomical convention.

An important concept when investigating visibility of sparkle is the contrast threshold at which luminous stimuli that are brighter than their surrounds become visible to observers. The most influential investigation on this topic was published in 1946 by Blackwell [21]. Prompted by its relevance for the visibility of military aviation and ship camouflage, more than two million responses were collected from "young women" observing an artificial circular stimulus against a darker background that acted as an adapting surround. The threshold for 50 percent probability of recognizing contrast was determined for different values of the surround's luminance and stimulus' luminance, which were called brightness by Blackwell, from the expression

$$
C_{\text {th }}=\left(L_{s}-L_{\text {surround }}\right) / L_{\text {surround }},
$$

where $C_{\text {th }}$ is the contrast threshold, and $L_{\text {surround }}$ and $L_{s}$ are the luminance of surround and stimulus, respectively.

The threshold data obtained by Blackwell were confirmed with better than 30\% accuracy by an independent perception study with luminous disks at the Naval Research
Laboratory [22], and by observations of star visibility during solar eclipses [23].

For determining the relevance of Blackwell's study for sparkle visibility, it should be noted that because of both optical diffraction and the retinal structure of the eye, stimuli subtending 1 arc min or less are known to appear as point sources [24]. Point sources follow Ricco's law [25], which establishes that the contrast threshold is inversely proportional to the solid angle subtended by the luminous stimulus:

$$
C_{\text {th }}=R\left(L_{\text {surround }}\right) / \omega_{s},
$$

where $R$ is a variable that depends only on the luminance of the surround, and $\omega_{s}$ is the solid angle subtended by the luminous stimulus.

By combining Eqs. (1) and (2), the stimulus' luminance required to observe it in a given surround luminance subtending a given small solid angle is obtained:

$$
L_{s}=L_{\text {surround }}\left(1+R\left(L_{\text {surround }}\right) / \omega_{s}\right) .
$$

In a very recent article [20], Crumey presented an empirical formula for the functional dependence of the variable $R$ on the surround luminance based on Blackwell's data:

$$
\begin{aligned}
& R(L)=\left(\sqrt{a_{1} L^{-1 / 2}+a_{2} L^{-1 / 4}+a_{3}}+a_{4} L^{-1 / 4}+a_{5}\right)^{2} \\
& a_{1}=5.949 \times 10^{-8}, \quad a_{2}=-2.389 \times 10^{-7}, \\
& a_{3}=2.459 \times 10^{-7}, \quad a_{4}=4.120 \times 10^{-4}, \\
& a_{5}=-4.225 \times 10^{-4} .
\end{aligned}
$$

Using Eqs. (3) and (4) it is possible to calculate the luminance a sparkle needs to have to be visible for the naked eye, as a function of distance, flake size, and surround luminance. From the calculated luminance it is also possible to estimate the photometric and geometrical characteristics of an illuminant in order to make sparkle visible for a specified size of metallic flake.

In later investigations, Hardy, Horman, and Clark used the results from Blackwell's study to predict the visibility of stars during daylight and in the not completely dark sky [26-28]. Depending on the position of the Sun just above or below the horizon, stars become just noticeable or not noticeable against the surrounding sky at some moment during dusk and dawn. This phenomenon is very similar to sparkle being just visible or not, depending on the luminance of the surrounding paint. However, it is important to note that, whereas stars are primary sources at different distances, sparkles are produced by flakes intervening as secondary light sources at similar distances. Therefore, astronomers are interested in the illuminance on earth emitted by stars at irrelevant distances, whereas in the sparkle problem we will assume that flakes are at very similar distances, and we will investigate the dependence of sparkle visibility on the lighting geometry.

From the data provided by Blackwell, after applying the correction proposed by Crumey for color temperature and psychophysical method [20], we found a very simple expression for the illuminance threshold:

$$
E_{50 \%}=L_{\text {surround }}^{0.788} \times 4.41 \times 10^{-8} \mathrm{~lm} / \mathrm{m}^{2} .
$$

The validity of this formula is restricted to surround luminances between 3 and $10,000 \mathrm{~lm} \mathrm{~m}^{-2} \mathrm{sr}^{-1}$, which is a range of 
values found in practical cases of sparkle observation (as further discussed below) and daylight observations of stars and planets. Rough indications for the luminance of sky regions at least $15 \mathrm{deg}$ from the Sun and from the horizon are up to 1000 and $5000 \mathrm{~lm} \cdot \mathrm{m}^{-2} \mathrm{sr}^{-1}$ in the case of a clear sky with the Sun being at least $5 \mathrm{deg}$ above the horizon $[18,22,27]$. These values decrease to $100 \mathrm{~lm} \cdot \mathrm{m}^{-2} \mathrm{sr}^{-1}$ for a heavily overcast day sky and to $1 \mathrm{~lm} \cdot \mathrm{m}^{-2} \mathrm{sr}^{-1}$ for a clear sky, $15 \mathrm{~min}$ after sunset $[\underline{18}, \underline{19}, \underline{27}, \underline{29}]$.

In Eq. (),,$E_{50 \%}$ is the illuminance threshold (unit: $1 \mathrm{~m} / \mathrm{m}^{2}$ ) of a point-like stimulus subtending 1 arc min or less, which is reported as visible by $50 \%$ of the observers against the surround. This equation produces almost the same result as Eq. (3), when it is converted into illuminance by multiplication with the solid angle subtended by the stimulus.

Hardy also discusses how empirical results from stellar visibility allow us to convert this threshold to a value for $98 \%$ visibility: this lowers the threshold in terms of stellar magnitude by a value of 0.75 . From Eq. ( $\underline{8})$ that is derived below, it is clear that this is equivalent to multiplying the threshold illuminance by $10^{0.75 / 2.5}=2.0[\underline{22}, \underline{26}]$. In this way we find

$$
E_{98 \%}=L_{\text {surround }}^{0.788} \times 8.82 \times 10^{-8} \mathrm{~lm} / \mathrm{m}^{2} .
$$

Another independent study was published by Knoll et al. [24]. An artificial bright stimulus of 1 arc min angular diameter was observed against a background field, for which the luminance was systematically varied. It was found that the "threshold for 100 percent seeing" could be expressed by the following relation:

$$
E_{100 \%}=c \times \sqrt{1+K L_{\text {surround }}} \mathrm{lm} / \mathrm{m}^{2} .
$$

Converted to modern units, the parameter values proposed by Knoll are equivalent to choosing $c=1.076 \times 10^{-9} \mathrm{~lm} \mathrm{~m}^{-2}$ and $K=10^{5} /\left(\mathrm{lm} \mathrm{m}^{-2} \mathrm{sr}^{-1}\right)[\underline{20}]$.

A numerical evaluation for Eqs. (ㅁ) $-(\underline{7})$ shows that the numerical values obtained with these equations are similar when applied to practical situations of sparkle visibility. This is also the case for the formula independently derived by Hecht [30], which is not further discussed here. For this reason, in the remainder of this article we will use Eq. (6).

\section{ASTRONOMICALLY BASED SPARKLE INTENSITY SCALE}

The analysis just provided predicts only if a sparkle is visible against its surround, but it does not quantify its perceived brightness. A quantitative scale of perceived sparkle intensity may be derived from our knowledge of perceived brightness of stars. The Hellenistic astronomer Hipparchos (150 BC) introduced a system of stellar magnitudes that accounts for the perceived brightness ("intensity") of star light. Hipparchos assigned a value of zero magnitude to the brightest star he could see (Vega), and values up to +6 to progressively less bright stars. In modern times stars brighter than Vega received negative values for their stellar magnitude. The brightest star, Sirius, which could not be observed from Hipparchos' observatory, has magnitude -1.4 . Stars less bright than magnitude 5 or 6 cannot be observed by the naked eye, with the threshold depending on atmospheric conditions and the presence of stray light.

The six levels of stellar magnitudes introduced by Hipparchos give an indication how the perceived brightness of stars scales with their illuminance. Stevens found that Hipparchos' stellar magnitude essentially follows a logarithmic scale in stellar illuminance as predicted by Fechner's law $[\underline{26}, \underline{31}]$ :

$$
m=2.5 \log _{10}\left(\frac{E_{0}}{E}\right) .
$$

Here, $m$ is the stellar magnitude, $E$ is the stellar illuminance, and $E_{0}=2.09 \times 10^{-6} \mathrm{~lm} / \mathrm{m}^{2}$ is the reference stellar illuminance [26].

When observing sparkle in metallic paints, the contrast between sparkle and the surrounding paint is almost never as large as it is for stars in the night sky. Therefore, the threshold for visually just perceivable stars, with a magnitude of +6 , gives a first estimate of the threshold for sparkle intensity of just noticeable sparkle: $8.32 \times 10^{-9} \mathrm{~lm} / \mathrm{m}^{2}$.

On the other hand, the brightest sparkles that can appear in a paint seem to be brighter than the brightest stars in the night sky. The planet Venus, which is often the brightest object in the sky (apart from the Sun and moon), can be as bright as magnitude -4.4, and even then it is less bright than the brightest sparkles that appear in dark metallic coatings on a sunny day. A crude lower estimate of the maximum sparkle intensity is therefore $1.2 \times 10^{-4} \mathrm{~lm} / \mathrm{m}^{2}$. A more accurate estimate will be derived in Section 4 .

The astronomical scale of stellar magnitudes indicates that the perceived sparkle intensity is expected to scale logarithmically with illuminance. This scale also makes it possible to estimate the just-noticeable difference for sparkle intensity. In the night sky, differences in stellar magnitude smaller than 1 unit can be distinguished. For example, in the constellation Gemini the difference in brightness between the stars Castor and Pollux, 0.5 units on the magnitude scale, is visually well recognizable. Recognizing differences in sparkle intensity is probably more difficult, because of the smaller contrast with surround.

These estimates can be used to obtain a first indication of the number of visually discriminable categories of sparkle intensity. With the sparkle intensity expected to range between stellar magnitude +6 and -4.4 , and a just-noticeable difference between 0.5 and 1 unit, we expect that 10 to 20 different categories of sparkle intensity can be visually distinguished. We note that in our previous work on sparkle, in which we did not specify sparkle intensity as a separate property, we showed that on an eight-point scale, observers' repeatability and reproducibility were found to be close to 0.5 units [1]. This suggests 17 different sparkle categories, thus confirming our preliminary analysis here.

\section{NUMERICAL EXAMPLES}

\section{A. Visibility of Sparkle in Direct Sunlight}

The luminance of the Sun as observed from sea level can be approximated by (see p. 28 in Ref. [32]) 
$L_{\text {sun }}=\left(1.87 \times 10^{9}\right) \times \exp \left(-0.209 / \sin \theta_{\text {sun }}\right) \operatorname{lm} /\left(\mathrm{m}^{2} \mathrm{sr}\right)$,

where $L_{\text {sun }}$ is the luminance of the Sun, and $\theta_{\text {sun }}$ is the angle between the Sun and the horizon.

For example, at a height of $30^{\circ}$ above the horizon, the solar luminance is $1.23 \times 10^{9} \mathrm{~lm} /\left(\mathrm{m}^{2} \mathrm{sr}\right)$. This luminance is concentrated on the solar disk, which has an angular diameter of $0.5 \mathrm{deg}$, corresponding to a solid angle of $5.98 \times 10^{-5} \mathrm{sr}$. By multiplying these quantities with each other we find the total illuminance of sunlight arriving at the surface of the observer's eye, including contributions originating from all parts of the solar disk. For the numerical example just given we find an illuminance of $7.37 \times 10^{4} \mathrm{~lm} / \mathrm{m}^{2}$.

Interestingly, when observing a sparkle formed by reflection of direct sunlight from a metallic flake, the flake acts a tiny mirror in the paint. Assuming 100\% reflectance of the aluminum flake, and assuming that the flake has the correct orientation for being observed as a sparkle, the flake represents a tiny but perfect mirror that allows us to see a very small part of the mirror image of the Sun. The luminance will then be equal to the luminance of the Sun. But because of the small size of the metallic flake, the illuminance of sparkle radiation incident in the eye of the observer is smaller than the illuminance from direct sunlight. For example, a flake with diameter $20 \mu \mathrm{m}$ observed from a distance of $30 \mathrm{~cm}$, and from an angle of $45^{\circ}$ with the flake normal, subtends a solid angle of $2.47 \times 10^{-9}$ sr. If it reflects light from the Sun when it is at $30^{\circ}$ above the horizon, the illuminance from the sparkle incident on the eye of the observer becomes $3.04 \mathrm{~lm} / \mathrm{m}^{2}$. This is considerably larger than the crude lower estimate that we calculated in the previous section.

According to Eq. (8), this corresponds to a stellar magnitude of -15.4 , which would make the sparkle brighter than the brightest planet or star. However, the sparkle is not observed against a dark night sky, but during the day under bright sunlight. Let us assume that the paint material surrounding the sparkle has a reflectance of $40 \%$. Let us also assume that it acts as a perfect Lambertian scatterer. Then the sunlight that is incident within an angular width of $5.98 \times 10^{-5} \mathrm{sr}$ is redirected by the paint material into a wide range of exit angles. After projection [19], this results in a luminance from the paint material surrounding the sparkle that can be calculated by multiplying the paint reflectance with the solar luminance and with the ratio between the solid angle subtended by the Sun and $\pi$. In this numerical example, we find a surround luminance of $9.38 \times 10^{3} \mathrm{~lm} /\left(\mathrm{m}^{2} \mathrm{sr}\right)$. There will also be a contribution to the surround luminance from reflection of other sky light. On a clear sunny sky, this ambient light also has a luminance up to $1 \times 10^{4} \mathrm{~lm} /\left(\mathrm{m}^{2} \mathrm{sr}\right)$, as we saw in the previous section. For a typical paint with refractive index of 1.5 , approximately $4 \%$ of this ambient light is directly reflected in the form of gloss, leading to a contribution of $400 \mathrm{~lm} /\left(\mathrm{m}^{2} \mathrm{sr}\right)$ to the surround luminance. In this example, this contribution is small.

The calculated total surround luminance is actually very bright, approximately as bright as the sky on a clear sunny day. For this value of surround luminance, Eq. (므) gives a $98 \%$ threshold for sparkle intensity (illuminance) of
$1.23 \times 10^{-4} \mathrm{~lm} / \mathrm{m}^{2}$. The calculated illuminance of the sparkle is clearly well above this threshold, so the sparkle is indeed observed as sparkle.

Based on this analysis, sparkle intensity (illuminance) and surround luminance for observing a metallic coating under direct sunlight can be calculated as

$$
\begin{aligned}
E_{\text {sparkle }}= & \left(1.87 \times 10^{9}\right) \frac{\pi D_{\text {flake }}^{2}}{4 d_{\text {obs }}^{2}} \cos \theta_{\text {obs }} \\
& \times \exp \left(-0.209 / \sin \theta_{\text {sun }}\right) 1 \mathrm{~m} /\left(\mathrm{m}^{2}\right), \\
L_{\text {surround }}= & \rho_{\text {gloss }} L_{\text {ambient }}+\frac{\rho_{\text {paint }} \omega_{\text {sun }}}{\pi}\left(1.87 \times 10^{9}\right) \\
& \times \exp \left(-\frac{0.209}{\sin \theta_{\text {sun }}}\right) \operatorname{lm} /\left(\mathrm{m}^{2} \mathrm{sr}\right),
\end{aligned}
$$

where $E_{\text {sparkle }}$ is the sparkle illuminance, $D_{\text {flake }}$ is the diameter of the flake, $\theta_{\text {obs }}$ is the angle of observation with respect to flake normal, $\rho_{\text {paint }}$ is the paint reflectance, and $d_{\text {obs }}$ is the observation distance. Further, $\omega_{\text {sun }}$ is the solid angle subtended by the Sun, $\rho_{\text {gloss }}$ is the fraction of incident light directly reflected as gloss, whereas $L_{\text {surround }}$ and $L_{\text {ambient }}$ are the luminances of the paint surrounding the sparkle and the ambient light, respectively. We note that from the second term in Eq. (11) the gloss reflection (typically $4 \%$ ) should be subtracted to be fully consistent.

By combining these expressions with the thresholds for visibility of sparkle as derived in Eqs. (56)-(7), we can estimate the smallest flake diameter that is visually distinguishable from the surround under direct sunlight and bright sky conditions. Figure 1 shows how this estimation varies with observation distance and paint reflectance. From this graph it is clear that under the given conditions, flakes as small as $1 \mu \mathrm{m}$ are predicted to be visible as sparkle from observation distances of a few meters or less. These predictions agree qualitatively well with the reported sparkle properties. For example, for highly reflective

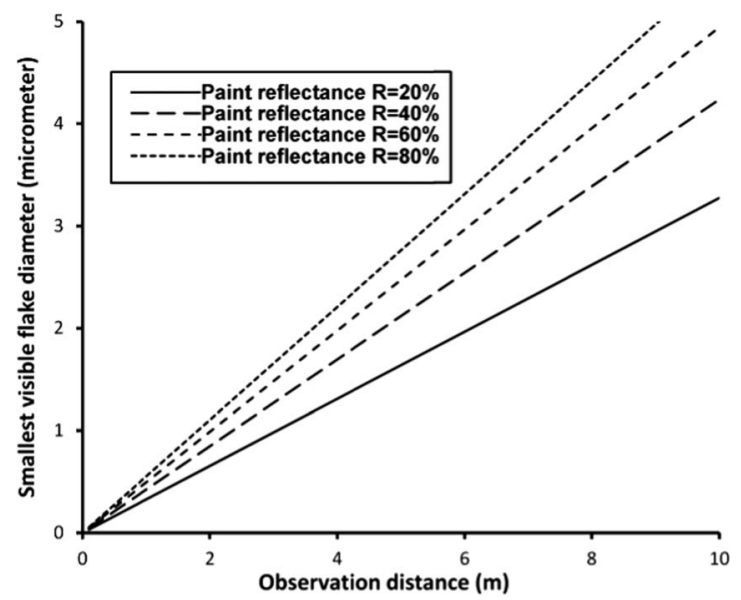

Fig. 1. Estimated threshold values for flake diameter, in order to be visible as sparkle, as a function of observation distance and paint reflectivity. Estimation based on Eqs. (10) and (11), for direct sunlight and bright sky conditions. Visual thresholds estimated with Eq. (6). Paint reflectance refers to parameter $\rho_{\text {paint }}$. 
flake pigments it has been reported that even pigment grades with the smallest average flake diameter that was investigated $(11 \mu \mathrm{m})$ give rise to intense sparkle [3].

Equations (10) and (11) also enable us to refine the crude estimation from Section $\underline{3}$ on the largest sparkle intensity (illuminance) that can be reached with common metallic coatings under practical lighting conditions. For the value of the flake diameter we use the largest value found in the automotive industry, which is about $30 \mu \mathrm{m}$. When viewing from only $20 \mathrm{~cm}$ distance, from an angle of $0 \mathrm{deg}$ with respect to the flake normal and with the Sun at its highest position in the sky, we calculate a sparkle intensity of $26.8 \mathrm{~lm} / \mathrm{m}^{2}$, corresponding to a stellar magnitude of -17.8 . This is indeed brighter than any star or planet in the night sky, as mentioned above.

\section{B. Visibility of Sparkle Under Artificial Spot Light}

The analysis just presented for viewing under direct sunlight can obviously be extended to viewing conditions with artificial spot light. As an example, we investigate the case of a metallic paint that is being examined with a highly intense spotlight, inside a body shop with common lighting switched on.

Measurements show that handheld spotlights in body shops typically deliver an illuminance of $12,000 \mathrm{~lm} / \mathrm{m}^{2}$ on the paint surface. With an aperture diameter of $10 \mathrm{~cm}$, operated at $30 \mathrm{~cm}$ lighting distance from the paint surface, this results in a solid angle of $0.086 \mathrm{sr}$ with respect to the paint surface. By calculating the ratio between illuminance and solid angle we find that in this example the luminance of the spotlight is $1.39 \times 10^{5} \mathrm{~lm} /\left(\mathrm{m}^{2} \mathrm{sr}\right)$.

By observing sparkle, the painter effectively looks at the mirror image of the spot light. Similar to the calculation in the previous example, we calculate that for a $20 \mu \mathrm{m}$ diameter flake observed from $30 \mathrm{~cm}$ distance under $45^{\circ}$ viewing angle, the sparkle intensity (illuminance) becomes $3.43 \times 10^{-4} \mathrm{~lm} / \mathrm{m}^{2}$. We note that even with this highly intense spotlight, the sparkle intensity (illuminance) is much smaller than the value we calculated for direct sunlight.

The luminance of the surround is calculated in a way similar to that in the previous section, using the paint reflectance and the illuminance and solid angle of the spot light. Diffuse scattering of light from the spotlight inside the paint medium is then found to contribute $1.53 \times 10^{3} \mathrm{~lm} /\left(\mathrm{m}^{2} \mathrm{sr}\right)$. The ambient lighting in a body shop working place is typically 1000 lux. Through direct reflection (gloss) from the paint, we get a contribution to the luminance of the surround, which, in this example, is estimated to be $6.4 \mathrm{~lm} /\left(\mathrm{m}^{2} \mathrm{sr}\right)$. The total luminance of the surround is therefore estimated to be $1.53 \times$ $10^{3} \mathrm{~lm} /\left(\mathrm{m}^{2} \mathrm{sr}\right)$. For this case, with Eq. (ㅁ) we calculate a $98 \%$ threshold for sparkle intensity (illuminance) of $2.86 \times 10^{-5} \mathrm{~lm} / \mathrm{m}^{2}$. The calculated sparkle intensity (illuminance) is again larger than this threshold value.

If we repeat this calculation for smaller flake diameters, we find that for a flake diameter of $5.7 \mu \mathrm{m}$, the $98 \%$ discriminability threshold is reached. The $50 \%$ threshold is already reached for a flake diameter of $4.0 \mu \mathrm{m}$. These values make clear why, for the finest grades of metallic coatings, for which average flake diameters smaller than $10 \mu \mathrm{m}$ are very common, hardly any sparkle can be observed when using spot light.
This analysis can be generalized for calculating sparkle intensity (illuminance) and surround luminance under spotlight as follows:

$$
\begin{gathered}
E_{\text {sparkle }}=\frac{E_{\text {spotlight }}}{\sin ^{2}\left(\frac{D_{\text {spotight }}}{2 d_{\text {spotight }}}\right)} \frac{D_{\text {flake }}^{2}}{4 d_{\text {obs }}^{2}} \cos \theta_{\text {obs }} 1 \mathrm{~m} /\left(\mathrm{m}^{2}\right), \\
L_{\text {surround }}=\frac{\rho_{\text {gloss }} E_{\text {ambient }}}{2 \pi}+\frac{\rho_{\text {paint }} E_{\text {spotlight }}}{\pi} \mathrm{lm} /\left(\mathrm{m}^{2} \mathrm{sr}\right),
\end{gathered}
$$

where $E_{\text {ambient }}$ is the illuminance of the ambient lighting, whereas $E_{\text {spotlight }}$ is the illuminance delivered by the spotlight as operated from a distance $d_{\text {spotlight }}$ from the paint surface. Further, $D_{\text {spotlight }}$ is the aperture diameter of the spotlight.

We have applied these equations for the artificial lighting conditions just described. When observing under those conditions a high gloss paint (i.e., gloss reflectance fixed at 0.04 ) of varying paint reflectance factor, observed under $45^{\circ}$ from several observation distances, then Eqs. (12), (13), and (6) combine to produce the estimations shown in Fig. 2 . For dark paints observed from very close distance, with paint reflectance factor 0.2 and observation distance $0.3 \mathrm{~m}$, the estimations show that metallic flakes of $5 \mu \mathrm{m}$ diameter or larger should be observable.

For lighter paints and/or larger observation distances, the smallest flakes that are observable have a diameter that is predicted to be as large as 10 or $20 \mu \mathrm{m}$. For example, from $3 \mathrm{~m}$ observation distance, only flakes larger than $30 \mu \mathrm{m}$ are predicted to be visible as sparkle. In metallic coatings, such large flakes are only rarely found. These estimations are confirmed by common experience with metallic coatings, in which sparkles generated by a spot light are not visible from observation distances larger than around a meter. When the same

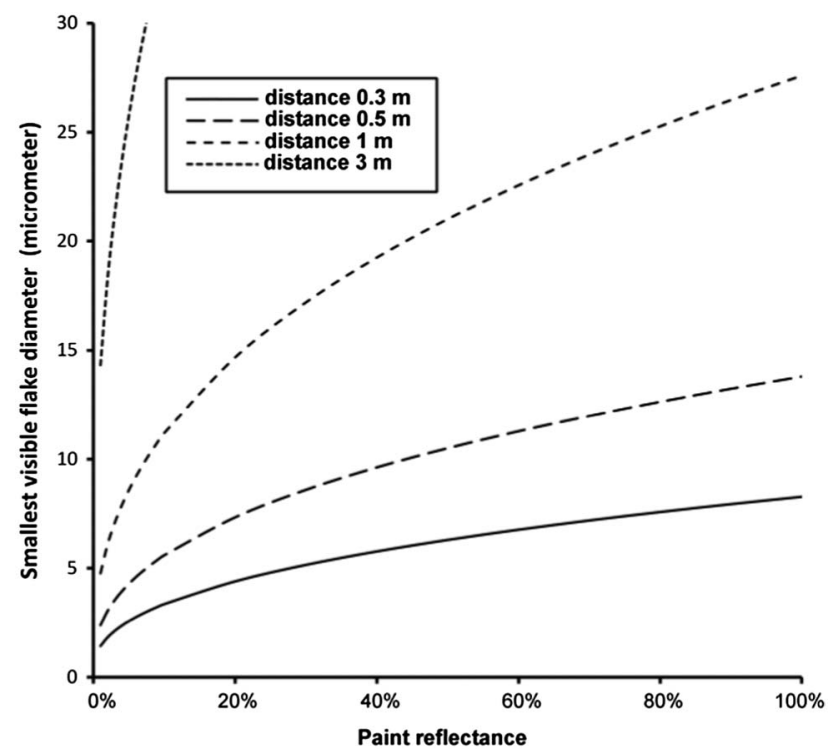

Fig. 2. Estimated threshold values for flake diameter, in order to be visible as sparkle, as a function of paint reflectance. Estimations are based on Eqs. (12) and (13), for artificial spot light conditions. Visual thresholds estimated with Eq. (6). Paint reflectance refers to parameter $\rho_{\text {paint }}$. 


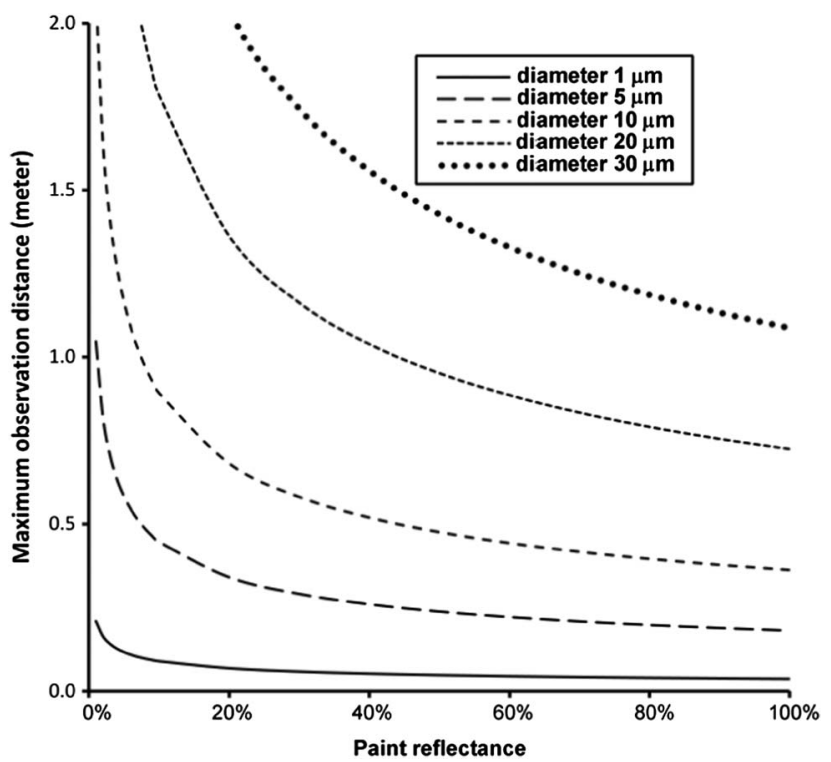

Fig. 3. Estimated threshold values for the maximum observation distance from which flakes with a specified diameter are observable as sparkle, as a function of paint reflectance. Estimations are based on Eqs. (12) and (13), for artificial spot light conditions. Visual thresholds estimated with Eq. (6). Paint reflectance refers to parameter $\rho_{\text {paint }}$.

metallic coatings are observed under direct sunlight, the sparkles are still visible at such distances, as correctly estimated with Eqs. (ㅁ),$(\underline{10})$, and (11).

In Fig. 3 , the estimations are presented in a different way. For a specified flake diameter, this graph shows the maximum distance at which the flake is observable as sparkle, as a function of paint reflectance. For example, flakes of $5 \mu \mathrm{m}$ diameter are predicted to only be visible as sparkle if the surrounding paint is dark enough and observed from distances close enough: the paint reflectance factor needs to be smaller than 0.2 and the observation distance needs to be smaller than $0.3 \mathrm{~m}$. In the same paint medium, flakes of $20 \mu \mathrm{m}$ diameter are predicted to be visible as sparkle also at observation distances up to $1.3 \mathrm{~m}$.

\section{COMPARING SPARKLE WITH STARS AND PLANETS}

We have already pointed out some similarities between observing sparkle in metallic coatings and observing stars and planets in the sky. As mentioned above, the brightness of the planet Venus seems similar to the maximum brightness that is obtained for sparkles in a metallic paint. In this section, we will further investigate these similarities.

The planet Venus has a physical diameter of approximately $12.100 \mathrm{~km}$. Observed from the surface of Earth, it is brightest when its distance to Earth is about 200 million kilometers. At that point, Venus has an angular diameter of $3.5 \times 10^{-3} \mathrm{deg}$. In comparison, a large metallic flake has a diameter of typically $20 \mu \mathrm{m}$, and it is observed from a distance of typically $30 \mathrm{~cm}$. In that case, we calculate an angular diameter of $3.8 \times 10^{-3} \mathrm{deg}$. This example shows that typical angular diameters of flakes in metallic coatings are very similar to the angular diameters of planets in the sky.
A star like Sirius, on the other hand, has a diameter of $2.8 \times$ $10^{6} \mathrm{~km}$ ( 2 times the diameter of the Sun) and a distance to Earth of $8.5 \times 10^{13} \mathrm{~km}$ (nine light years). Its angular diameter is therefore $1.9 \times 10^{-6} \mathrm{deg}$. This is much smaller than the angular diameter encountered when observing sparkle. For metallic flakes of $20 \mu \mathrm{m}$ diameter, the same angular diameter as Sirius would be obtained when observing the paint from $600 \mathrm{~m}$ distance. At that distance, sparkles are no longer visible.

We conclude that under normal viewing distances of less than a meter, the visibility of sparkle has much in common with the observation of planets, even more than with observing stars.

A final remark needs to be made regarding eye accommodation, because it may influence observations. In the case of sparkle, the eye is adapted to a photoptic level according to the background luminance, and accommodated to a more or less short observation distance. In the case of observing stars during the night, the eye is adapted to darkness and not accommodated. When observing stars and planets during daylight or twilight conditions, the eye is adapted to the corresponding background luminance. It remains to be investigated if the eye adaptation and accommodation levels for that case are comparable to the situation when sparkle is being observed.

\section{CONCLUSIONS}

We have seen that when investigating sparkle intensity of metallic coatings, there is much to be learned from the perception literature, including work related to astronomical observations of stars and especially planets. We derived thresholds for just-noticeable sparkles based on the existing literature. These thresholds were shown to depend critically on the reflectance of the paint medium surrounding the metallic flakes inside the metallic paint.

In numerical examples, we showed how the magnitude of sparkle intensity may be calculated from physical properties such as flake diameter, observation distance, illuminance of the light source, and reflection properties of the paint. We provided mathematical equations that can be used to calculate if flake reflections lead to a perceived sparkle effect. Based on this analysis, we predict that the sparkle intensity (illuminance) of visually distinguishable sparkles may vary from $2.1 \times 10^{-6}$ to $26.8 \mathrm{~lm} / \mathrm{m}^{2}$. Depending on circumstances, the actual range of sparkle intensity is part of this range.

The calculations show that under appropriate illumination and observation conditions, metallic flakes of only $1 \mu \mathrm{m}$ diameter can cause sparkle to be seen. However, we also showed why under typical situations with an intense spot light, sparkle is not seen in fine metallic coatings: under such conditions, only flakes with diameters exceeding 5-7 $\mu \mathrm{m}$ can give rise to sparkle. These predictions agree with common observations of sparkle in metallic coatings.

Finally, we have indicated how to calculate the number of categories of sparkle intensity that can be visually distinguished for given illumination and observation conditions. Also, this prediction is confirmed by earlier observations of metallic sparkle.

In the derivations, we made several assumptions related to the visual adaptation state and resolving power of the eye, the 
dependence on wavelength, and the point-like character of sparkles. The analysis given here would benefit from a future examination of these topics.

European Metrology Research Programme (EMRP) Spanish Ministry of Economy and Competitiveness(DPI201130090-C02) Comunidad de Madrid (CM: S2013/MIT-2790).

The EMRP is jointly funded by the EMRP participating countries within the European Association of National Meteorlogy (EURAMET) and the European Union.

\section{REFERENCES}

1. E. J. J. Kirchner, G. J. van den Kieboom, S. L. Njo, R. Supèr, and R. Gottenbos, "The appearance of metallic and pearlescent materials," Color Res. Appl. 32, 256-266 (2007).

2. J. Patzlaff and M. Rösler, "Sparkle effects in thin layers," Eur. Coat. J. 01-02, 56-57 (2006).

3. T. Rentschler, "Measuring sparkling blues without blues," Eur. Coat. J. 12, 78-83 (2011).

4. R. Schmid, C. Lavallee, S. A. Jones, and J. Carroll, "Patent application," U.S. patent 0237683A1 (January 2, 2011).

5. C. Schmidt and X. Petsitis, "Interference effect pigments-new technologies in cosmetic products," SÖFW J. 136, 42-48 (2010).

6. P. Wissling, Metallic Effect Pigments (Vincentz, 2006).

7. G. A. Klein, Industrial Color Physics (Springer, 2010).

8. A. Jacobsen and A. Gilchrist, "The ratio principle holds over a millionto-one range of illumination," Percept. Psychophys. 43, 1-6 (1988).

9. S. Kitaguchi, S. Westland, M. R. Luo, E. J. J. Kirchner, and G. J. van den Kieboom, "Application of HDR imaging to modeling of glints in metallic coatings," in Proceedings of the Interim Meeting of the International Color Association (AIC, 2008).

10. Z. Huang, H. Xu, and M. R. Luo, "Camera-based model to predict the total difference between effect coatings under directional illumination," Chin. Opt. Lett. 9, 093301 (2011).

11. A. Ferrero, J. Campos, A. M. Rabal, and A. Pons, "A single analytical model for sparkle and graininess patterns in texture of effect coatings," Opt. Express 21, 26812-26819 (2013).

12. I. B. N. van der Lans, E. Kirchner, and A. Half, "Accurate appearancebased visualization of car paints," in Proceedings of the European Conference on Colour in Graphics, Imaging, and Vision (The Society for Imaging Science and Technology, 2012), pp. 17-23.
13. R. W. Preisendorfer, "Secchi disk science: visual optics of natural waters," Limnol. Oceanogr. 31, 909-926 (1986).

14. J. Koenderink, "Trieste in the mirror," Perception 29, 127-133 (2000).

15. C. Cox and W. Munk, "Measurement of the roughness of the sea surface from photographs of the sun's glitter," J. Opt. Soc. Am. 44, 838-850 (1954).

16. S. Kay, J. Hedley, and S. Lavender, "Sun glint estimation in marine satellite images: a comparison of results from calculation and radiative transfer modeling," Appl. Opt. 52, 5631-5639 (2013).

17. A. Ryer, Light Measurement Handbook (International Light, 1997).

18. K. Tohsing, M. Schrempf, S. Riechelmann, H. Schilke, and G. Seckmeyer, "Measuring high-resolution sky luminance distributions with a CCD camera," Appl. Opt. 52, 1564-1573 (2013).

19. J. M. Palmer and B. G. Grant, The Art of Radiometry (SPIE, 2010).

20. A. Crumey, "Human contrast threshold and astronomical visibility," Mon. Not. R. Astron. Soc. 442, 2600-2619 (2014).

21. H. R. Blackwell, "Contrast thresholds of the human eye," J. Opt. Sci. Am. 36, 624-643 (1946).

22. R. Tousey and E. O. Hulburt, "The visibility of stars in the daylight sky," J. Opt. Soc. Am. 38, 886-896 (1948).

23. G. P. Können and C. Hinz, "Visibility of stars, halos and rainbows during solar eclipses," Appl. Opt. 47, H14-H24 (2008).

24. H. A. Knoll, R. Tousey, and E. O. Hulburt, "Visual thresholds of steady point sources of light in fields of brightness from dark to daylight," J. Opt. Soc. Am. 36, 480-482 (1946).

25. A. Ricco, "Relazione fra il minimo angolo visual e l'intensita luminosa," Ann. d'Ottalmol. 6, 373-479 (1877).

26. A. C. Hardy, "How large is a point source?" J. Opt. Soc. Am 57, 44-47 (1967).

27. M. H. Horman, "Visibility of light sources against a background of uniform luminance," J. Opt. Soc. Am 57, 1516-1521 (1967).

28. R. N. Clark, Visual Astronomy of the Deep Sky (Cambridge University, 1990).

29. J. I. Gordon, C. F. Edgerton, and S. Q. Duntley, "Signal-light nomogram," J. Opt. Soc. Am. 65, 111-118 (1975).

30. S. Hecht, "Visual thresholds of steady point sources of light in fields of brightness from dark to daylight," J. Opt. Soc. Am. 37, 59 (1947).

31. S. S. Stevens, Psychophysics: Introduction to Its Perceptual, Neural and Social Aspects (Wiley, 1975).

32. G. Wyszecki and W. S. Stiles, Color Science: Concepts and Methods, Quantitative Data and Formulae (Wiley, 2000). 